\title{
Importance of colonoscopy in patients undergoing endoscopic resection for superficial esophageal squamous cell carcinoma
}

\author{
Kei Tominaga ${ }^{a}$, Hisashi Doyama ${ }^{a}$, Hiroyoshi Nakanishia, Naohiro Yoshidaa, Yasuhito Takedaa, \\ Ryosuke Ota ${ }^{a}$, Kunihiro Tsuji ${ }^{a}$, Kazuhiro Matsunaga ${ }^{a}$, Shigetsugu Tsujia, Kenichi Takemura ${ }^{a}$, \\ Shinya Yamada ${ }^{a}$, Kazuyoshi Katayanagi ${ }^{b}$, Hiroshi Kurumaya ${ }^{b}$
}

Ishikawa Prefectural Central Hospital, Kanazawa, Japan

Abstract

\begin{abstract}
Background The aim of the study was to clarify the frequency of colorectal neoplasm (CRN) complicating superficial esophageal squamous cell carcinoma (ESCC) and the need for colonoscopy.
\end{abstract}
Methods We retrospectively reviewed 101 patients who had undergone initial endoscopic resection (ER) for superficial ESCC. Control group participants were age- and sex-matched asymptomatic subjects screened at our hospital over the same period of time. Advanced adenoma was defined as an adenoma $\geq 10 \mathrm{~mm}$, with villous features, or high-grade dysplasia. Advanced CRN referred to advanced adenoma or cancer. We measured the incidence of advanced CRN in superficial ESCC and controls, and we compared the characteristics of superficial ESCC patients with and without advanced CRN.

Results In the superficial ESCC group, advanced CRNs were found in 17 patients (16.8\%). A history of smoking alone was found to be a significant risk factor of advanced CRN [odds ratio 6.02 (95\% CI 1.30-27.8), $\mathrm{P}=0.005$ ].

Conclusion The frequency of synchronous advanced CRN is high in superficial ESCC patients subjected to ER. Colonoscopy should be highly considered for most patients who undergo ER for superficial ESCC with a history of smoking, and is recommended even in superficial ESCC patients.

Keywords Advanced adenoma, colonoscopy, colorectal neoplasm, esophageal squamous cell carcinoma

Ann Gastroenterol 2016; 29 (2): 1-7

\section{Introduction}

According to the statistical analysis conducted by the International Agency for Research on Cancer under the auspices of the World Health Organization (WHO) [1], both

Departments of ${ }^{\mathrm{a}}$ Gastroenterology (Kei Tominaga, Hisashi Doyama, Hiroyoshi Nakanishi, Naohiro Yoshida, Yasuhito Takeda, Ryosuke Ota, Kunihiro Tsuji, Kazuhiro Matsunaga, Shigetsugu Tsuji, Kenichi

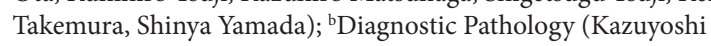
Katayanagi, Hiroshi Kurumaya), Ishikawa Prefectural Central Hospital, Kanazawa, Japan

\section{Conflict of Interest: None}

Correspondence to: Kei Tominaga, MD, Department of Gastroenterology, Ishikawa Prefectural Central Hospital, 2-1 Kuratsuki-Higashi, Kanazawa city, Ishikawa, Japan, Tel.: +81 762378211 , Fax: +81 762385366 , e-mail: keipoo9004@yahoo.co.jp, tominaga@ipch.jp

Received 29 December 2015; accepted 9 February 2016

DOI: http://dx.doi.org/10.20524/aog.2016.0025 the incidence and mortality of esophageal cancer are high, making it one of the major malignancies on a worldwide basis. This is especially true in the so-called 'esophageal cancer belt' extending from Iran via Central Asia to China. Esophageal cancer has been noted to be more common in developing countries than in developed ones, and to occur 2-4 times more often in men than in women. As for histologic type, in contrast to Western countries, in China, Taiwan, and Japan esophageal squamous cell carcinoma (ESCC) is the most common, and particularly in Japan exceeds $90 \%$ of cases [2,3]. Alcohol consumption and smoking have been recognized as risk factors for ESCC.

Esophageal cancer is known to frequently overlap with other cancers, notably those of the head and neck, lung, and stomach [4-6]. ESCC is particularly prone to coexist with cancers of the head and neck, and stomach, and there is broad recognition that attention needs to be paid to these sites at the time of surveillance.

Esophageal cancer is considered to bear an unfavorable prognosis, although with the development of better endoscopes and radiological equipment and advances in therapeutic 
techniques, detection at an early stage and an increase in the proportion of treated cases have been achieved [7]. For these reasons, long-term survival is now achievable in many cases, and reflecting the increase in the number of long-term survival cases [8,9], the detection of overlapping second and third primary cancers has also been increasing [4-6,10]. Sites of second and third primary cancers developing in these patients include head/neck, stomach, and lung [4-6], while in recent years, colorectal neoplasm (CRN) also frequents coexists [10-13], and thus colonoscopy is recommended in this patient group. In particular, Baeg et al [12] have argued that since CRN is frequently detected both before and after therapy for ESCC, colonoscopy should be considered when ESCC is first diagnosed. However, these reports focused on esophageal adenocarcinoma [10], and, even when addressing ESCC, they concentrated on advanced tumors [11,13] with much less attention devoted to the frequency of CRN coexisting with superficial ESCC or the need for colonoscopy in this context. Furthermore, esophageal cancer and colon cancer are known to share common risk factors such as male sex, smoking, and heavy alcohol consumption [14-16].

The present study aimed to clarify the frequency of CRN coexisting with superficial ESCC and the need for colonoscopy at the time of diagnosis of superficial ESCC, by determining the details of alcohol consumption and smoking history from superficial ESCC patients who had undergone initial endoscopic resection (ER) as related to the overlap with CRN.

\section{Patients and methods}

\section{Study design and patients}

We retrospectively reviewed a total of consecutive 336 patients, who had undergone ER for superficial ESCC between August 2006 and October 2014 at our hospital. Excluding 98 patients undergoing ER for any asynchronous lesions, we focused solely on the 238 patients present at the initial therapy. ER was indicated for ESCC satisfying principally the following condition: depth of tumor within T1b-SM1 regardless of size, as determined by preoperative endoscopy. Baseline characteristics and endoscopic and histopathological data were reviewed from the medical records. We excluded 6 patients who had a past history of colorectal cancer, 56 patients who had not undergone colonoscopy within one year before or after ER for superficial ESCC, 41 patients for whom the details regarding alcohol consumption and/or smoking history were unclear, and 34 patients for whom we could not find age- and sex-matched controls, leaving 101 patients suitable for the final analysis (Fig. 1). Almost all superficial ESCC patients were asymptomatic and had undergone a colonoscopy due to screening. Control group participants were selected from asymptomatic subjects at the medical examination for screening in our hospital at the same time and were matched to the patients by age and sex. Patients with a past history of colorectal cancer were also excluded.

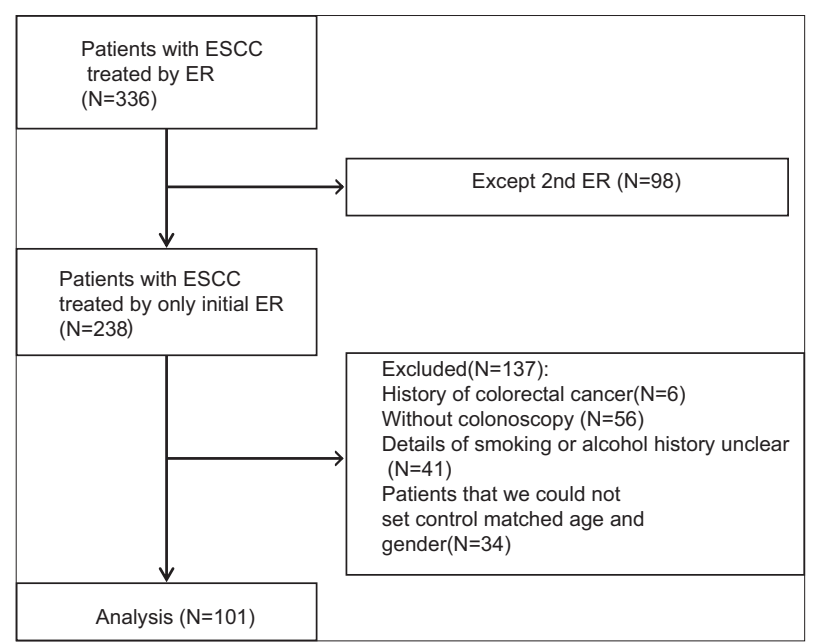

Figure 1 Flowchart of the review and analysis

ESCC, esophageal squamous cell carcinoma; ER, endoscopic resection

This observational study was conducted at a single tertiary referral center in Japan, as part of the Strengthening the Reporting of Observational Studies in Epidemiology (STROBE) program [17]. In accordance with the Declaration of Helsinki, the institutional review board of Ishikawa Prefectural Central Hospital approved this study, and written informed consent was obtained from all subjects or legal guardians.

\section{Histology and definition of ESCC}

The postoperative histopathological diagnosis was made by two board-certified pathologists of the Japanese Society of Pathology (K.K., H.K.). The diagnoses were made with reference to the revised Vienna classification [18] and WHO classification [19]. According to the WHO classification [19] high-grade intraepithelial neoplasia (HGIN) is clinically important because HGINs have the potential to become malignant invasive cancers [20]. For the purposes of this study, we defined the revised Vienna categories 4 and 5 as ESCC.

\section{Colonoscopy procedure}

In the present investigation, the day of treatment of a superficial ESCC was regarded as the point of reference, and the findings of colonoscopy performed within one year before or after it were adopted. The endoscope-videoscope system used was EVIS LUCERA SPECTRUM system (Olympus Co., Tokyo, Japan) or EVIS LUCERA ELITE system (Olympus Co., Tokyo, Japan). The endoscope used was PCF-240ZI (Olympus Co., Tokyo, Japan), PCF-Q260AZI (Olympus Co., Tokyo, Japan), or CF-H260AZI (Olympus Co., Tokyo, Japan). All colonoscopic examinations were performed by experienced endoscopists after routine preparation including 2-4 L of polyethylene glycol or 1-2 L of polyethylene glycol plus ascorbate solution.

To facilitate the identification and diagnosis of colonic lesions, in addition to white light observation, magnifying 
chromoendoscopy or magnifying endoscopy with narrowband imaging (M-NBI) was also used. Diagnosis according to magnifying chromoendoscopy was based on the pit pattern classification of Kudo, Tsuruta et al [21], and that according to M-NBI was based on Sano's capillary classification [22]. CRN was judged to be present when a IIIs, IIIL, IV, or V pit pattern (in Kudo's classification), or a Type II, IIIA, or IIIB (in Sano's classification) was evident. Lesions suspected of being $\geq 5 \mathrm{~mm}$ CRN were subjected to ER or surgical resection, and were then examined histologically. Even if lesions were suspected of being $<5 \mathrm{~mm} \mathrm{CRN}$, they were subjected to ER and then examined histologically when they showed IV, V on the pit pattern classification or Type IIIA, IIIB on Sano's classification. Other lesions were observed conservatively, and, even though resected endoscopically, they were not sent for histological examination.

\section{Definition of advanced adenoma and advanced CRN}

Histopathological diagnosis was performed by two boardcertified pathologists of the Japanese Society of Pathology (K.K., H.K.). The diagnoses of resected CRN were made with reference to the revised Vienna classification [18]. For the purposes of this study, we defined the revised Vienna categories 3 and 4 as adenoma and the revised Vienna classification category 5 as cancer. Lesions suspected of being CRN but not subjected to histological evaluation were counted as adenomas when they showed IIIs, IIIL on the pit pattern classification and Type II on Sano's capillary classification. An advanced adenoma was defined as a revised Vienna category 3 lesion $\geq 10 \mathrm{~mm}$, with villous features or revised Vienna category 4 lesion. Advanced CRN referred to advanced adenoma or cancer.

\section{Outcome measurements}

Outcomes were: 1) the incidence of advanced CRN in superficial ESCC patients and controls; 2) characteristics of superficial ESCC patients with and without advanced CRN. Both were compared with respect to age, sex, body mass index (BMI), tumor size, depth, site, presence/absence of synchronous multiple superficial ESCC, underlying disease(s), aspirin use, statin use, alcohol consumption history, smoking history, cancer family history (esophageal cancer, colon cancer, other cancer), and personal history of cancer.

\section{Statistical analysis}

When synchronous lesions were detected at the time of the initial therapy for superficial ESCC, the respective largest lesion size and greatest depth were adopted for the present analyses. Data are shown as the prevalence or median value. In comparisons of superficial ESCC patients and controls, continuous variables were analyzed with the Wilcoxon signed- rank test, and categorical variables were compared using the McNemar test, as appropriate. $\mathrm{P}<0.05$ was considered significant. In comparisons of superficial ESCC patients with and without advanced CRN, continuous variables were analyzed with the Mann-Whitney $U$ test, and categorical variables were compared using the Chi-square test or Fisher's exact test, as appropriate. Statistical analysis was performed using IBM SPSS version 20 (SPSS Inc., Tokyo, Japan).

\section{Results}

Baseline epidemiological characteristics of the 101 patients with superficial ESCC and controls are listed in Table 1. Median age was 67 years and there was a male predilection. Median tumor size of the ESCC was $19 \mathrm{~mm}$, and depth was T1a-epithelium/lamina propria mucosa in $80.2 \%$. Tumors were located most frequently in the middle intrathoracic esophagus, and synchronous lesions were noted in 19 cases (18.8\%). There were no differences observed in BMI, diabetes mellitus, statin and aspirin intake. In superficial ESCC patients

Table 1 Baseline characteristics of the superficial ESCC patients and controls

\begin{tabular}{|c|c|c|c|}
\hline & $\begin{array}{c}\text { ESCC } \\
(\mathrm{N}=101)\end{array}$ & $\begin{array}{l}\text { Controls } \\
(\mathrm{N}=101)\end{array}$ & $\mathrm{P}$ value \\
\hline Median age (years; range) & $67(42-86)$ & $67(42-86)$ & $>0.99$ \\
\hline Sex (men/women) & $83 / 18$ & $83 / 18$ & $>0.99$ \\
\hline $\operatorname{BMI}\left(<25 / \geq 25, \mathrm{~kg} / \mathrm{m}^{2}\right)$ & $82 / 19$ & $71 / 30$ & 0.10 \\
\hline \multicolumn{4}{|l|}{ Underlying disease (s) } \\
\hline Diabetes mellitus, (\%) & $16(15.8 \%)$ & $26(25.7 \%)$ & 0.08 \\
\hline Cardiac disease, $(\%)$ & $6(5.9 \%)$ & $5(5.0 \%)$ & 0.76 \\
\hline Cerebrovascular disease, $(\%)$ & $5(5.0 \%)$ & $4(4.0 \%)$ & 0.73 \\
\hline Aspirin intake, $(\%)$ & $2(2.0 \%)$ & $2(2.0 \%)$ & $>0.99$ \\
\hline Statin intake, (\%) & $17(16.8 \%)$ & $12(11.9 \%)$ & 0.32 \\
\hline $\begin{array}{l}\text { Alcoholic beverages: ethanol } \\
(<60 / \geq 60 \text {, g/day })\end{array}$ & $81 / 20$ & $95 / 6$ & 0.006 \\
\hline Smoking: pack years $(<20 / \geq 20)$ & $36 / 65$ & $60 / 41$ & 0.001 \\
\hline \multicolumn{4}{|l|}{ Cancer family history } \\
\hline Esophageal cancer, (\%) & $7(6.9 \%)$ & $5(5.0 \%)$ & 0.55 \\
\hline Colorectal cancer, $(\%)$ & $5(5.0 \%)$ & $7(6.9 \%)$ & 0.55 \\
\hline Other cancers, $(\%)$ & $58(57.4 \%)$ & $46(45.5 \%)$ & 0.09 \\
\hline $\begin{array}{l}\text { Personal cancer, (\%) } \\
\text { (some overlap) }\end{array}$ & $31(30.7 \%)$ & $14(13.9 \%)$ & 0.02 \\
\hline Stomach & 17 & 6 & \\
\hline Pharyngolarynx & 8 & 1 & \\
\hline Lung & 3 & 2 & \\
\hline Liver & 2 & 0 & \\
\hline Other & 4 & 7 & \\
\hline
\end{tabular}


the alcohol consumption history and the smoking history were significantly higher than controls. The cancer family history was similar between superficial ESCC patients and controls. Excluding esophageal cancer and colon cancer, stomach cancer at a rate of $16.8 \%$ was the most frequently noted in the personal

Table 2 Incidence of advanced CRN in superficial ESCC patients and controls

ESCC Controls $\mathrm{P}$ value $(\mathrm{N}=101) \quad(\mathrm{N}=101)$

\begin{tabular}{lccc}
\hline Advanced CRN & $17(16.8 \%)$ & $6(5.9 \%)$ & 0.025 \\
Advanced adenoma (some overlap) & $16(15.8 \%)$ & $5(5.0 \%)$ & \\
\hline Low-grade adenoma $\geq 10 \mathrm{~mm}$ in size & 7 & 4 \\
With villous features & 0 & 0 \\
High-grade dysplasia & 10 & 1 \\
Cancer & $1(1.0 \%)$ & $1(1.0 \%)$ \\
\hline
\end{tabular}

cancer history followed by pharyngolaryngeal cancer at a rate of $7.9 \%$, both being more frequent than the rates observed in controls (5.9\% and $1.0 \%, \mathrm{P}=0.02$, respectively).

The results of colonoscopy in the 101 patients of superficial ESCC and 101 controls are listed in Table 2. Advanced CRNs were found in 17 of the 101 superficial ESCC patients (16.8\%), compared with $6(5.9 \%)$ in controls $(\mathrm{P}=0.025)$. Advanced adenomas were present in 16 cases (15.8\%), consisting of lowgrade adenomas $\geq 10 \mathrm{~mm}$ in size in 7 patients, those with villous features in 0 patients, high-grade dysplasia in 10. Cancer was detected in 1 patient (1.0\%). In 16 of 17 patients (94.1\%) ER was performed. The remaining 1 patient showed advanced cancer, for which surgical curative resection was undertaken.

The 17 patients with superficial ESCC and advanced CRNs and the 84 patients with superficial ESCC without advanced CRNs were compared with respect to age, sex, body mass index (BMI), tumor size, depth, site, presence/absence of synchronous multiple superficial ESCC, underlying disease(s), aspirin use, statin use, alcohol consumption history, smoking

Table 3 Characteristics of superficial ESCC patients with and without advanced CRN

\begin{tabular}{|c|c|c|c|}
\hline & $\begin{array}{l}\text { With advanced } \\
\text { CRN }(\mathrm{N}=17)\end{array}$ & $\begin{array}{l}\text { Without advanced } \\
\text { CRN }(\mathrm{N}=84)\end{array}$ & $\mathrm{P}$ value \\
\hline Median age (years; range) & $68(55-75)$ & $66(42-86)$ & 0.66 \\
\hline Gender (men/women) & $16 / 1$ & $67 / 17$ & 0.29 \\
\hline $\operatorname{BMI}\left(<25 / \geq 25, \mathrm{~kg} / \mathrm{m}^{2}\right)$ & $13 / 4$ & $69 / 15$ & 0.73 \\
\hline ESCC tumor size (mm, median; range) & $21(10-60)$ & $18(2-67)$ & 0.13 \\
\hline ESCC tumor depth $(\mathrm{EP} \bullet \mathrm{LPM} / \mathrm{MM} \bullet \mathrm{SM} 1 / \mathrm{SM} 2)$ & $13 / 2 / 2$ & $68 / 13 / 3$ & 0.14 \\
\hline ESCC tumor location (upper/middle/lower) & $0 / 13 / 4$ & $2 / 71 / 11$ & 0.14 \\
\hline ESCC number of synchronous ESCC (solitary/multiple) & $13 / 4$ & $69 / 15$ & 0.73 \\
\hline \multicolumn{4}{|l|}{ Underlying disease $(s)$} \\
\hline Diabetes mellitus, (\%) & $0(0 \%)$ & $16(19.0 \%)$ & 0.07 \\
\hline Cardiac disease, $(\%)$ & $0(0 \%)$ & $6(7.1 \%)$ & 0.59 \\
\hline Cerebrovascular disease, $(\%)$ & $0(0 \%)$ & $5(6.0 \%)$ & 0.59 \\
\hline Aspirin intake, $(\%)$ & $0(0 \%)$ & $2(2.4 \%)$ & $>0.99$ \\
\hline Statin intake, $(\%)$ & $2(11.8 \%)$ & $15(17.9 \%)$ & 0.73 \\
\hline Alcoholic beverages: ethanol $(<60 / \geq 60$, g/day $)$ & $12 / 5$ & $69 / 15$ & 0.32 \\
\hline Smoking: pack years $(<20 / \geq 20)$ & $1 / 16$ & $35 / 49$ & 0.005 \\
\hline \multicolumn{4}{|l|}{ Cancer family history } \\
\hline Esophageal cancer, $(\%)$ & $1(5.9 \%)$ & $6(7.1 \%)$ & $>0.99$ \\
\hline Colorectal cancer, $(\%)$ & $0(0 \%)$ & $5(6.0 \%)$ & 0.59 \\
\hline Other cancers, $(\%)$ & $9(52.9 \%)$ & $49(58.3 \%)$ & 0.79 \\
\hline Personal cancer, (\%) (some overlap) & $7(41.2 \%)$ & $21(25.0 \%)$ & 0.23 \\
\hline Stomach & 3 & 14 & \\
\hline Pharyngolarynx & 1 & 6 & \\
\hline Lung & 2 & 1 & \\
\hline Liver & 1 & 1 & \\
\hline Other & 2 & 2 & \\
\hline
\end{tabular}

ESCC, esophageal squamous cell carcinoma; CRN, colorectal neoplasm; BMI, body mass index; EP, epithelium; LPM, lamina propria mucosa; MM, muscularis mucosa; SM, submucosa 
history, cancer family history (esophageal cancer, colon cancer, other cancer), and personal history of cancer, respectively (Table 3). A history of smoking alone was found to be a significant risk factor for advanced CRNs [odds ratio $6.02(95 \%$ CI 1.30-27.8), $\mathrm{P}=0.005]$.

\section{Discussion}

Superficial ESCC before and after therapy is known to often coincide with primary cancers at other sites, notably the head and neck, stomach, and lung $[5,6,10,11]$. The association of CRN has mostly been described with advanced stage ESCC, and even though the proportion of advanced CRN varies widely with reported extremes of $7-66.7 \%$, its frequency is undoubtedly high $[12,13]$. However, no reports are available concerning CRN in superficial ESCC limited to cases treated with ER, which prompted us to investigate whether similar to the early stage of esophageal cancer the frequency of CRN would be equally high. In our study of 101 superficial ESCC patients subjected to colonoscopy, advanced adenoma was detected in $15.8 \%$ and cancer in $1.0 \%$, accounting for a total of $16.8 \%$ of advanced CRNs. In this way a high proportion of such lesions was detected consistent with the results of previous studies that focused on the advanced stage of ESCC. Moreover and notably, the majority of these advanced CRNs were lesions for which cure could be anticipated with ER.

Frequently co-existent pharyngolaryngeal cancer, gastric cancer and lung cancer can be easily detected during the preoperative workup of esophageal cancer by upper gastrointestinal endoscopy and CT examinations. In contrast, the preparation required for colonoscopy to detect colon cancer is onerous for the patient, and in the presence of advanced age and other conditions there may be considerable hesitation to impose it on the patient. As a consequence, at our institution the implementation rate of colonoscopy at about one year before and after therapy in ER cases for superficial ESCC has been inadequate at only $76.5 \%$. On the other hand, perhaps because of differences in the recognition of the association of advanced CRNs, the implementation rate of colonoscopy for advanced stage ESCC is high at 95\% [14]. These results suggest that in ER for superficial ESCC as well, a search for synchronous colon cancer would be recommended.

With regard to colon cancer, the removal of colonic adenomas has been reported to lower the risk of colorectal cancer [23]. Previous studies have reported that certain colonoscopic findings, including adenoma size $\geq 1 \mathrm{~cm}$, highgrade dysplasia, villous histology, are associated with an increased risk of metachronous advanced neoplasm [24-27]. When these high-risk findings are present, follow up by colonoscopy is recommended, and in the present work we defined these high-risk findings as advanced adenoma, and considered advanced adenoma together with colon cancer as advanced CRN.

Although the epidemiological and pathophysiological associations between ESCC and colorectal cancer remain unclear, the two diseases share some common risk factors such as male sex, smoking, heavy alcohol consumption, and high intake of red meat $[15-17,28]$. They also share some common prophylactic interventions such as the use of aspirin and statins $[29,30]$. In addition, previous studies have reported a positive association between age, BMI and risk of CRN [31,32]. In our ESCC group with advanced CRNs, however, no significant differences were noted in almost any of the factors investigated such as age, sex, BMI, heavy alcohol consumption, diabetes mellitus, or aspirin or statin use, with a smoking history alone being identified as a significant finding.

Colorectal cancer was added to the list of smokingattributable cancers by the International Association for Research on Cancer in 2009 [33,34] and by the US Surgeon General in 2014 [35]. According to several recent metaanalyses, the relationship between smoking and colorectal cancer incidence is likely dose dependent [36-39]. Furthermore, recent cohort study reported that smoking is associated with a nearly two-fold higher risk of death compared with never smoking [40]. Smoking was also positively associated with colorectal cancer-specific mortality. However, the biologic mechanisms underlying this association are not well understood. Smoking has been associated with risk of specific molecular phenotypes of colorectal cancer,

\section{Summary Box}

\section{What is already known:}

- Esophageal cancer is known to frequently overlap with other cancers, notably those of the head and neck, lung, and stomach

- Coexistence of esophageal cancer with colorectal neoplasms (CRN) has been frequently reported, and thus colonoscopy is recommended in this patient group

- The frequency of CRN coexisting with superficial esophageal squamous cell carcinoma (ESCC) or the need for colonoscopy are not well established yet

\section{What the new findings are:}

- Advanced CRNs were found in 17 of the 101 superficial ESCC patients (16.8\%), and there were significantly more in them than controls $(5.9 \%$, $\mathrm{P}=0.025$ )

- The frequency of synchronous advanced CRN is high even in superficial ESCC subjected to endoscopic resection

- At our institution, the implementation rate of colonoscopy at about one year before and after therapy in endoscopic resection cases for superficial ESCC has been inadequate at only $76.5 \%$ 
including tumors characterized as microsatellite instability high [41-44] or CIMP high [44-46] or with BRAF mutations [43-45]. The impact of smoking on colorectal cancer survival differs according to tumor molecular phenotype, although the patterns of association across tumor molecular phenotypes varied and were not well known. Regarding the amount of smoking, it was extremely high in the advanced CRN group at smoking $\geq 20$ pack years. However, in 106 smoking patients with 20 pack years and more, advanced CRN were found 16 cases of 65 superficial ESCC patients (24.6\%), and 2 cases of 41 controls (4.9\%). Regardless of smoking, it can be said that ESCC might itself be a risk factor for advanced CRN.

Limitations of the present study include the fact that it was retrospective in nature, and was conducted at a single institution. In addition, not all of the superficial ESCC patients subjected to ER underwent a colonoscopy and got the details of alcohol consumption and smoking history. The analyzed patients were not examined about information on tumor molecular phenotype or intake of red meat. Also, additional investigation will be needed to define the long-term course of advanced CRN after ER for superficial ESCC.

In conclusion, the frequency of synchronous advanced CRN is high even in superficial ESCC subjected to ER. Colonoscopy should be highly considered for most patients who undergo ER for superficial ESCC with a history of smoking, and would be recommended in even superficial ESCC patients.

\section{References}

1. Ferlay J, Soerjomataram I, Dikshit R, et al. Cancer incidence and mortality worldwide: sources, methods and major patterns in GLOBOCAN 2012. Int J Cancer 2015;136:E359-E386.

2. Lin Y, Totsuka Y, He Y, et al. Epidemiology of esophageal cancer in Japan and China. J Epidemiol 2013;23:233-242.

3. Lin MY, Chen MC, Wu IC, et al. Areca users in combination with tobacco and alcohol use are associated with younger age of diagnosed esophageal cancer in Taiwanese men. PLoS One 2011;6:e25347.

4. Nandy N, Dasanu CA. Incidence of second primary malignancies in patients with esophageal cancer: a comprehensive review. Curr Med Res Opin 2013;29:1055-1065.

5. Kumagai Y, Kuwano T, Nakajima Y, et al. Multiple primary cancers associated with esophageal carcinoma. Surg Today 2001;31:872-876.

6. Matsubara T, Yamada K, Nakagawa A. Risk of second primary malignancy after esophagectomy for squamous cell carcinoma of the thoracic esophagus. J Clin Oncol 2003;21:4336-4341.

7. Natsugoe $S$, Baba M, Yoshinaka H, et al. Mucosal squamous cell carcinoma of the esophagus: a clinicopathologic study of 30 cases. Oncology 1998;55:235-241.

8. Kato $\mathrm{H}$, Tachimori $\mathrm{Y}$, Watanabe $\mathrm{H}$, Yamaguchi $\mathrm{H}$, Ishikawa $\mathrm{T}$, Itabashi M. Superficial esophageal carcinoma: surgical treatment and results. Cancer 1990;66:2139-2323.

9. Nishimaki T, Suzuki T, Suzuki S, Kuwabara S, Hatakeyama K. Outcomes of extended radical esophagectomy for thoracic esophageal cancer. J Am Coll Surg 1998;186:306-312.

10. Bollschweiler E, Schloesser T, Leers J, Vallböhmer D, Schäfer $\mathrm{H}$, Hölscher AH. High prevalence of colonic polyps in white males with esophageal adenocarcinoma. Dis Colon Rectum 2009;52:299-304.
11. Poon RT, Law SY, Chu KM, Branicki FJ, Wong J. Multiple primary cancers in esophageal squamous cell carcinoma: incidence and implications. Ann Thorac Surg 1998;65:1529-1534.

12. Baeg MK, Choi MG, Jung YD, et al. Esophageal squamous cell carcinoma patients have an increased risk of coexisting colorectal neoplasm. Gut Liver 2015; 17:60-66.

13. Takeuchi D, Koide N, Komatsu D, Suzuki A, Miyagawa S. Prevalence and management of colorectal neoplasia in surgically treated esophageal cancer patients. Int J Surg 2015;17:60-66.

14. Anderson JC, Alpern Z, Sethi G, et al. Prevalence and risk of colorectal neoplasia in consumers of alcohol in a screening population. Am J Gastroenterol 2005;100:2045-2055.

15. Anderson JC, Attam R, Alpern Z, et al. Prevalence of colorectal neoplasia in smokers. Am J Gastroenterol 2003;98:2777-2783.

16. Rabeneck L Paszat LF, Hilsden RJ, et al. Advanced proximal neoplasia of the colon in average-risk adults. Gastrointest Endosc 2014;80:660-667.

17. von Elm E, Altman DG, Egger M, Pocock SJ, Gøtzsche PC, Vandenbroucke JP; STROBE Initiative. Strengthening the reporting of observational studies in epidemiology (STROBE) statement: guidelines for reporting observational studies. BMJ 2007;335:806-808.

18. Schlemper RJ, Riddell RH, Kato Y, et al. The Vienna classification of gastrointestinal epithelial neoplasia. Gut 2000;47:251-225.

19. Gabbert HE, Shimoda T, Hainaut P, Nakamura Y, Field JK, Inoue H. WHO classification of tumors: pathology and genetics of tumors of the digestive system. IARC Press: Lyon (France); 2000:9-19.

20. Takubo K, Aiba J, Sawabe M, et al. Early squamous cell carcinoma of the oesophagus: the Japanese viewpoint. Histopathology 2007;51:733-742.

21. Kudo S, Hirota S, Nakajima T, et al. Colorectal tumors and pit pattern. J Clin Pathol 1994;47:880-885.

22. Uraoka T, Saito Y, Ikematsu H, Yamamoto K, Sano Y. Sano's capillary classification for narrow band imaging of early colorectal lesions. Dig Endosc 2011;23(Suppl 1): 112-115.

23. Zanber AG, Winawer SJ, O’Brien MJ, et al. Colonoscopic polypectomy and long-term prevention of colorectal-cancer deaths. N Engl J Med 2012;366:687-696.

24. Winawer SJ, Zauber AG, Fletcher RH, et al. Guidelines for colonoscopy surveillance after polypectomy: a consensus update by the US Multi-Society Task Force on Colorectal Cancer and the American Cancer Society. Gastroenterology 2006;130:1872-1885.

25. Davila RE, Rajan E, Baron TH, et al. ASGE guideline: colorectal cancer screening and surveillance. Gastrointest Endosc 2006;63:546-557.

26. Arditi C, Peytremann-Bridevaux I, Brnand B, et al. Appropriateness of colonoscopy in Europe (EPAGE II): screening for colorectal cancer. Endoscopy 2009;41:200-208.

27. Lee SM, Kim JH, Sung IK, Hong SN. The risk of metachronous advanced colorectal neoplasia rises in parallel with an increasing number of high-risk findings at baseline. Gut Liver 2015;23:741-749.

28. Qu X, Ben Q, Jiang Y. Consumption of red and processed meat and risk for esophageal squamous cell carcinoma based on a metaanalysis. Ann Epidemiol 2013;23:762-770.

29. Sandler RS, Halabi S, Baron JA, et al. A randomized trial of aspirin to prevent colorectal adenomas in patients with previous colorectal cancer. N Engl J Med 2003;348:883-890.

30. Alexandre L, Clark AB, Bhutta HY, Holt S, Lewis MP, Hart AR. Statin use is associated with reduced risk of histologic subtypes of esophageal cancer: a nested case-control analysis. Gastroenterology 2014;146:661-668.

31. Diamond SJ, Enestvedt BK, Jiang Z, et al. Adenoma detection rate increase with each decade of life after 50 years of age. Gastrointest Endosc 2011;74:135-140.

32. Stein B, Anderson JC, Rajapakse R, Alpern ZA, Messina CR, Walker G. Body mass index as a predictor of colorectal 
neoplasia in ethnically diverse screening population. Dig Dis Sci 2010;55:2945-2952.

33. Secretan B, Straif K, Baan R, et al. A review of human carcinogens: Pert E: Tobacco, areca nut, alcohol coal smoke, and salted fish. Lancet Oncol 2009;10:1033-1034.

34. International Agency for Research on Cancer: IARC monograph on the evaluation of carcinogenic risks to humans. International Agency for Research on Cancer, Lyon, France, 2012.

35. US Department of Health and Human Services. The health consequences of smoking: 50 years of progress. A report of the surgeon general. Centers for Disease Control and Prevention, National Center for Chronic Disease Prevention and Health Promotion, Office on Smoking and Health, Atlanta, GA, 2014.

36. Tsoi KK, Pau CY, Wu WK, Chan FK, Griffiths S, Sung JJ. Cigarette smoking and the risk of colorectal cancer: a metaanalysis of prospective cohort studies. Clin Gastroenterol Hepatol 2009;7:682-688.

37. Huxley RR, Ansary-Moghaddam A, Clifton P, Czemichow S, Parr CL, Woodward M. The impact of dietary and lifestyle risk factors on risk of colorectal cancer: a quantitative overview of the epidemiological evidence. Int J Cancer 2009;125:171-180.

38. Liang PS, Chen TY, Giovannucci E. Cigarette smoking and colorectal cancer incidence and mortality: systematic review and meta-analysis. Int J Cancer 2009;124:2406-2415.

39. Botteri E, Iodice S, Bagnardi V, Raimondi S, Lowenfels AB,
Maisonneuve P. Smoking and colorectal cancer: a meta-analysis. JAMA 2008;300:2765-2778.

40. Yang B, Jacobs EJ, Gapstur SM, Stevens V, Campbell PT. Active smoking and mortality among colorectal cancer survivors: the Cancer Prevention Study II nutrition cohort. J Clin Oncol 2015;33:885-893.

41. Slattery ML, Curtin K, Anderson K, et al. Associations between cigarette smoking, lifestyle factors, and microsatellite instability in colon tumors. J Natl Cancer Inst 2000;92:1831-1836.

42. Poynter JN, Haile RW, Siegmund KD, et al. Association between smoking, alcohol consumption, and colorectal cancer, overall and by tumor microsatellite instability status. Cancer Epidemiol Biomarkers Prev 2009;18:2745-2750.

43. Curtin K, Samowitz WS, Wolff RK, Herrick J, Caan BJ, Slattery ML. Somatic alterations, metabolizing genes and smoking in rectal cancer. Int J Cancer 2009;125:158-164.

44. Limsui D, Virkant RA, Tillmans LS, et al. Cigarette smoking and colorectal cancer risk by molecularly defined subtypes. I Natl Cancer Inst 2010;102:1012-1022.

45. Samowitz WS, Albertsen H, Sweeney C, et al. Association of smoking, CpG island methylator phenotype, and V600E BRAF mutations in colon cancer. J Natl Cancer Inst 2006;98:1731-1738.

46. Nishihara R, Morikawa T, Kuchiba A, et al. A prospective study of duration of smoking cessation and colorectal cancer risk by epigenetics-related tumor classification. Am J Epidemiol 2013; 178:84-100. 